\title{
Variability of magnetic field spectra in the Earth's magnetotail
}

\author{
A. A. Petrukovich and D. V. Malakhov \\ Space Research Institute, 84/32 Profsoyuznaya st., Moscow, 117997, Russia \\ Moscow Institute of Physics and Technology, Moscow, Russia
}

Received: 13 October 2009 - Revised: 1 December 2009 - Accepted: 2 December 2009 - Published: 15 December 2009

\begin{abstract}
We investigate the variability of magnetic fluctuation spectra below $1 \mathrm{~Hz}$ in the Earth's plasma sheet using specially selected long observation intervals by Geotail spacecraft. The spectra can be generally described by a negative power law with two kinks. The range between kinks $\sim 0.02-0.2 \mathrm{~Hz}$ has the most stable power law index $\sim 2.4-2.6$. Indices at the lower and the higher frequencies are more variable and generally increase with power of fluctuations. In the sub-second range fluctuations are strongly localized and indices are closer to 3 . At the lower-frequency end indices are about 1.5 . The lower kink is usually well defined on average spectra and its frequency tends to increase with activity. Combination of spectrum index $\alpha$ and fractal dimension $\delta$ is expected to follow the Berry relation $\alpha+2 \delta=5$, but actually is $\sim 5.5$.
\end{abstract}

\section{Introduction}

Magnetic fluctuations are in the focus of research in space plasma physics since the first spacecraft experiments. In the magnetotail plasma sheet special attention is given to frequency range below $\sim 1-10 \mathrm{~Hz}$, which contains the most powerful fluctuations. The lowest frequency $\sim 0.01-$ $0.001 \mathrm{~Hz}$ is actually limited by the duration of a data interval. Observations with IMP, ISEE, AMPTE, Geotail and other spacecraft revealed variety of magnetic wave phenomena (e.g., Russell, 1972; Gurnett et al., 1976; Michalov et al., 1970; Bauer et al., 1995; Borovsky and Funsten, 2003; Hoshino et al., 1994; Sigsbee et al., 2001). Magnetic power spectral density (PSD) generally follows a power law with respect to frequency: PSD $\sim f^{-\alpha}$. The power index $\alpha$ was $\sim 2-$ 2.5 in the range $\sim 20-200 \mathrm{mHz}$. At lower frequencies $\alpha$ was lower than $\sim 1.5$ and kinks in spectra were generally well defined at some frequency slightly above $0.01 \mathrm{~Hz}$. At the higher frequencies (around $1 \mathrm{~Hz}$ ) $\alpha$ was $\sim 3$. Fluctuations exhibited

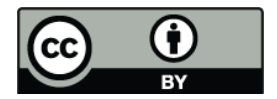

Correspondence to: A. A. Petrukovich (apetruko@iki.rssi.ru) no preference to right-hand/left-hand polarizations and compressible/incomressible modes. Waves were enhanced during substorms and high-speed flows. Recently with Cluster data these conclusions were generally confirmed (Volwerk et al., 2003, 2004; Vörös et al., 2004, 2007; Weygand et al., 2005). In addition dependence of PSD indices from plasma flow velocity was revealed (Vörös et al., 2007; Volwerk et al., 2004). In particular in the lower frequency part indices were about 1.5 only for sufficiently high fluctuation amplitudes related with plasma flows.

Power law frequency spectra are a characteristic signature of scale-invariant or fractal curves (Mandelbrot, 1977). The fractal dimension $\delta$ can be computed directly in the temporal domain. With Ampte-CCE spacecraft data magnetic $\delta$ for the time scales smaller than several seconds was $\sim 1.3$, while for the longer time scales it was $\sim 1.8$ and very variable (Ohtani et al., 1995, 1998).

A significant effort was also devoted to studies of nonGaussian properties of fluctuations (intermittency or multifractality) (e.g., Vörös et al., 2003; Weygand et al., 2005) but this aspect will not be addressed in the current investigation. A more detailed review of previous investigations can be found elsewhere (e.g., Petrukovich, 2005).

Theoretical efforts in understanding of fluctuations were initially directed to analysis of stability criteria and signatures of various wave modes. In the recent years another paradigm gained popularity. It is attempted to understand magnetotail dynamics focusing on collective statistical properties of variations (e.g., Chang, 1999; Chang et al., 2003; Zelenyi et al., 1998; Lui, 2002). Power indices thus could be compared with that of the Kolmogorov (5/3) or Kraichnan (3/2) turbulence cascades (e.g. Dudok de Wit and Krasnosel'skikh, 1996). Alternatively Milovanov and Zelenyi (Milovanov et al., 1996, 2001; Zelenyi et al., 1998) suggested that in a part of the studied frequency range magnetic fluctuations may appear as a fractal pattern with PSD index (7/3) due to self-consistent coupling of magnetic field with nonadiabatic ions, creating electric currents. This state could be understood as a non-linear saturation stage of a multitude of plasma wave modes.

Published by Copernicus Publications on behalf of the European Geosciences Union and the American Geophysical Union. 
However, despite general consistency of different observations, significant variance in results still exists. Theories mostly operate with spatial characteristics, which reveal itself as a time sequence of measurements, recorded by spacecraft. The magnetosphere is ever "breathing" due to external and internal reasons, and local variations or waves can have their own (phase) velocities. Characteristic time scales of substorms are of the order of one hour, while more local bursty plasma flows last several minutes. The simplest applicable approach is known as the Taylor hypothesis. It states that the intrinsic time-dependence of (frozen-in) fluctuations can be neglected as they are convected past the spacecraft by a plasma bulk flow (Dudok de Wit and Krasnosel'skikh, 1996; Borovsky and Funsten, 2003). However, in the plasma sheet with intrinsically uneven convection, exact correspondence between spatial and temporal scales will be likely smeared out. This problem substantially reduces possibilities of analysis as compared, e.g., with the solar wind case.

For collection of substantial statistics it is necessary to analyze several-hour-long intervals of more or less homogeneous fluctuations, but such cases are very rare in the magnetosphere. In this paper we analyze variability of spectral indices in such specially selected intervals.

It is convenient to define three frequency ranges with differing indices, selection of which is justified by combination of theoretical considerations and observational results. Boundary frequencies in what follows are approximate. The proton cyclotron frequency is $\sim 0.15 \mathrm{~Hz}$ for $10 \mathrm{nT}$.

1. LF: $(<0.02 \mathrm{~Hz})-$ the main sources of activity in this range are large-scale magnetotail flapping, dipolarizations, MHD eddies, etc. The lowest frequency is mostly limited by the maximal length of a data sample, and could be about $\sim 1 \mathrm{mHz}$.

2. MF: $(\sim 0.02-0.2 \mathrm{~Hz})-$ just below the proton cyclotron frequency. At these scales magnetic variations are coupled with non-adiabatic ion motion.

3. HF: $(>0.2 \mathrm{~Hz})-$ waves in this range are essentially local (generated by local gradients).

\section{Techniques of data analysis}

Geotail magnetic (1/16-s sampling) and ion (12-s sampling) measurements in the GSM coordinates were used for our analysis (Kokubun et al., 1994; Mukai et al., 1994). Geotail spacecraft due to its equatorial orbit, synchronized with the magnetotail seasonal up and down shift, is optimal for such a study. We selected seven intervals of long-duration plasma sheet observations (Table 1). Here the detailed analysis of indices for one interval (29 November 1996) is presented, while for the rest only average data are given. The primary sampling rate of the magnetic DC channel is $128 \mathrm{~Hz}$, then the signal is averaged onboard to 16 samples per second. Therefore at the frequencies close to the Nyquist frequency $(8 \mathrm{~Hz})$
Table 1. List of the intervals.

\begin{aligned} & \hline Date time, UT \\ & \hline 1 Feb 1996 $14: 00-22: 00 \\ &$ 7 Feb 1996 $17: 00-22: 00 \\ &$ 1 Oct 1996 $12: 00-14: 00 \\ &$ 29 Nov 1996 $12: 00-18: 00 \\ &$ 4 Feb 1998 $08: 00-16: 00 \\ &$ 2 Mar 2001 $12: 00-24: 00 \\ &$ 16 Apr 2005 $00: 00-08: 00 \\ &$\hline\end{aligned}

variations are attenuated and aliased. We actually used only frequencies below $1 \mathrm{~Hz}$, where this effect can be neglected.

Frequency spectra calculations were performed by the means of the fast Fourier transform (Bendat and Piersol, 1986), which advantages and limitations are well understood. The wavelet technique (e.g., Dudok de Wit, 2003; Vörös et al., 2004) helps to study more transient signals. However, since we are interested in multi-scale characteristics (spectral indices), some averaging of spectra at the smaller scales is anyway necessary, while required spatial resolution can be achieved with careful selection of interval length for Fourier analysis. The length spectrum (basically the first order structure function) of a signal can be computed as $L(\tau)=\sum\left|B\left(t_{k}+\tau\right)-B\left(t_{k}\right)\right|$, where $\tau$ is variable time step. For an ideal scale-invariant curve $L(\tau)=L_{0} \tau^{1-\delta}$. We followed Ohtani et al. (1995), using slightly different $L^{*}(\tau)$ with the additional $\tau$ in the denominator $\left(L^{*}(\tau) \sim \tau^{-\delta}\right)$ and the special coefficient, adjusting for jumps in $L^{*}(\tau)$, when $\tau$ is comparable with the total length. Indices were computed with the least squares fitting of log-log spectra by the first order polynomials as well as by the bilinear function with a kink (adopted from Hoshino et al., 1994). The latter were used mainly to locate spectral kinks.

A number of actions was undertaken to reduce possible errors during index determination:

1. Fourier spectra have linear frequency scale, therefore density of the points in the log scale is increasing towards high frequencies and this part may acquire disproportionate high statistical weight in fitting. Thus before fitting the spectra were resampled to a new logarithmically spaced frequency scale. However this effect actually did not contribute much to variance of indices.

2. Initially relatively long data samples were taken to determine kink frequencies. All conclusions about indices were later cross-checked using samples, optimized for the specific frequency range.

3. To exclude possibility of systematic algorithm errors, in a test case we analyzed the fractional Brownian curve with the fractal dimension close to that of our data. The error in $\alpha$ and $\delta$ computation was less than 0.02 , while their sum $\alpha+2 \delta$ was equal to 5.02 . 
Table 2. Average and variance of indices, kinks, for 11.29.96 on 16384-point samples.

\begin{tabular}{llllllll}
\hline Selection & $\alpha_{\mathrm{LF}}$ & $\delta_{\mathrm{LF}}$ & $\alpha_{\mathrm{LF}}+2 \delta_{\mathrm{LF}}$ & $\alpha_{\mathrm{MF}}$ & $\delta_{\mathrm{MF}}$ & $\alpha_{\mathrm{MF}}+2 \delta_{\mathrm{MF}}$ & kink, Hz \\
\hline $\mathrm{X}$ & $1.67 \pm 0.68$ & $2.30 \pm 0.56$ & 6.27 & $2.47 \pm 0.36$ & $1.58 \pm 0.10$ & 5.63 & $0.045 \pm 0.02$ \\
$\mathrm{Y}$ & $1.28 \pm 0.70$ & $2.11 \pm 0.28$ & 5.50 & $2.25 \pm 0.46$ & $1.64 \pm 0.09$ & 5.53 & $0.024 \pm 0.02$ \\
$\mathrm{Z}$ & $1.35 \pm 0.62$ & $1.89 \pm 0.14$ & 5.13 & $2.44 \pm 0.45$ & $1.56 \pm 0.14$ & 5.56 & $0.029 \pm 0.02$ \\
\hline
\end{tabular}

Such a sum $\alpha+2 \delta=5$ (Berry relation) is often used in theory and data analysis to convert indices between temporal and frequency domains (Mandelbrot, 1977; Burlaga and Klein, 1986; Abry et al., 2000). We also check applicability of this expression to our data set.

Finally it is important to mention, that values of indices may also depend on the type of averaging of PSD spectra, if signal power is substantially varying and index changes with power. Two types of averaging generally can be applied: averaging over neighboring frequencies after taking a spectrum on a single (long) interval and averaging of spectra after dividing the initial interval in several samples. The latter sample averaging is a sum of squared amplitudes with the preference to higher-power parts, while the spectrum, taken on a longer interval, is a sum of amplitudes, less selective in terms of power. Similarly the average index can be computed using the average spectrum (preference to higher power due to square averaging) or taking the mean of indices of individual spectra (independent from power).

\section{Data description and statistics}

The 6-h long crossing of inner plasma sheet $\left(\left|B_{x}\right|\right.$ was generally within $10 \mathrm{nT}$ ), which occurred on 29 November 1996, is well suited for our statistical analysis (Fig. 1). It was a period of enhanced magnetospheric convection with series of fast plasma flows, happening during moderately southward IMF (not shown here). Negative bays at the nightside auroral zone stations were within 300-400 nT (not shown here). Magnetic field variations on the scales of minutes and tens of minutes were strong and relatively even throughout the interval. However, variations on the scale of seconds (the 3-s RMS panel in Fig. 1) were very spiky.

Sample PSD and length spectra of $B_{z}$ computed for the 16384-point (approximately $1000 \mathrm{~s}$ ) interval starting at 12:17 UT, are in Fig. 2. PSD spectra were smoothed with a moving average. The slope of the PSD spectrum is rather variable, and with selection of appropriate frequency range almost any value of index could be retrieved. The PSD spectrum flattens above approximately $2 \mathrm{~Hz}$, where the signal power becomes comparable with the instrument noise. The length spectrum (Fig. 2b) is much smoother due to averaging of many individual differences at each scale.
Table 3. Average and variance of indices for 11.29 .96 for adjusted samples.

\begin{tabular}{|c|c|c|c|}
\hline Selection & $\alpha$ & $\delta$ & $\alpha+2 \delta$ \\
\hline \multicolumn{4}{|c|}{ LF on 65536-point samples } \\
\hline $\mathrm{X}$ & $1.85 \pm 0.42$ & $1.84 \pm 0.14$ & 5.53 \\
\hline Y & $1.52 \pm 0.11$ & $1.92 \pm 0.07$ & 5.36 \\
\hline $\mathrm{Z}$ & $1.26 \pm 0.20$ & $1.89 \pm 0.03$ & 5.04 \\
\hline \multicolumn{4}{|c|}{ MF on 4096-point samples } \\
\hline $\mathrm{X}$ & $2.49 \pm 0.46$ & $1.47 \pm 0.10$ & 5.43 \\
\hline Y & $2.42 \pm 0.58$ & $1.52 \pm 0.13$ & 5.45 \\
\hline $\mathrm{Z}$ & $2.53 \pm 0.36$ & $1.52 \pm 0.12$ & 5.57 \\
\hline \multicolumn{4}{|c|}{ HF on 1024-point samples } \\
\hline $\mathrm{X}$ & $2.78 \pm 0.51$ & $1.38 \pm 0.10$ & 5.54 \\
\hline $\mathrm{Y}$ & $2.88 \pm 0.63$ & $1.40 \pm 0.11$ & 5.68 \\
\hline $\mathrm{Z}$ & $2.83 \pm 0.62$ & $1.38 \pm 0.12$ & 5.59 \\
\hline
\end{tabular}

The whole event was initially divided in $21 \times 16384$-point samples. For each sample PSD and length spectra were computed, then bilinear functions were fitted in the ranges 0.004$0.2 \mathrm{~Hz}$ and $0.02-2 \mathrm{~Hz}$. Each fit returned four values: kink frequency, two indices and power at the kink. However such sample length turned out to be not optimal for some parameters of interest. In particular upper kink frequencies and HF indices were poorly defined (not shown here). Indices for the LF and the MF ranges (mean values and variance) and the corresponding kink frequencies are in Table 2. In some individual samples of the LF range $\delta$ values were larger than 2 (even the average in Table 2 is more than 2). The fits may return such numbers due to, e.g., insufficient statistics on large scales.

Therefore for more precise determination of LF indices $5 \times 65538$-point samples were used (Table 3 ). All $\delta$-s now were within 2 , while $\alpha$ were $1.2-1.8$, essentially the same as for the 16384-point samples. Also, in the longer intervals power and velocity variations were averaged out almost completely. Therefore the further PSD analysis was performed still with the 16384-point spectra. We looked for correlations between fit parameters and plasma characteristics. Correlation between the power and the LF index is evident, the spectra were steeper for higher power (Fig. 3a). The linear regression coefficient between the decimal logarithm of power (in $\mathrm{nT}^{2} / \mathrm{Hz}$ ) and $\alpha$ was 1.1 . 


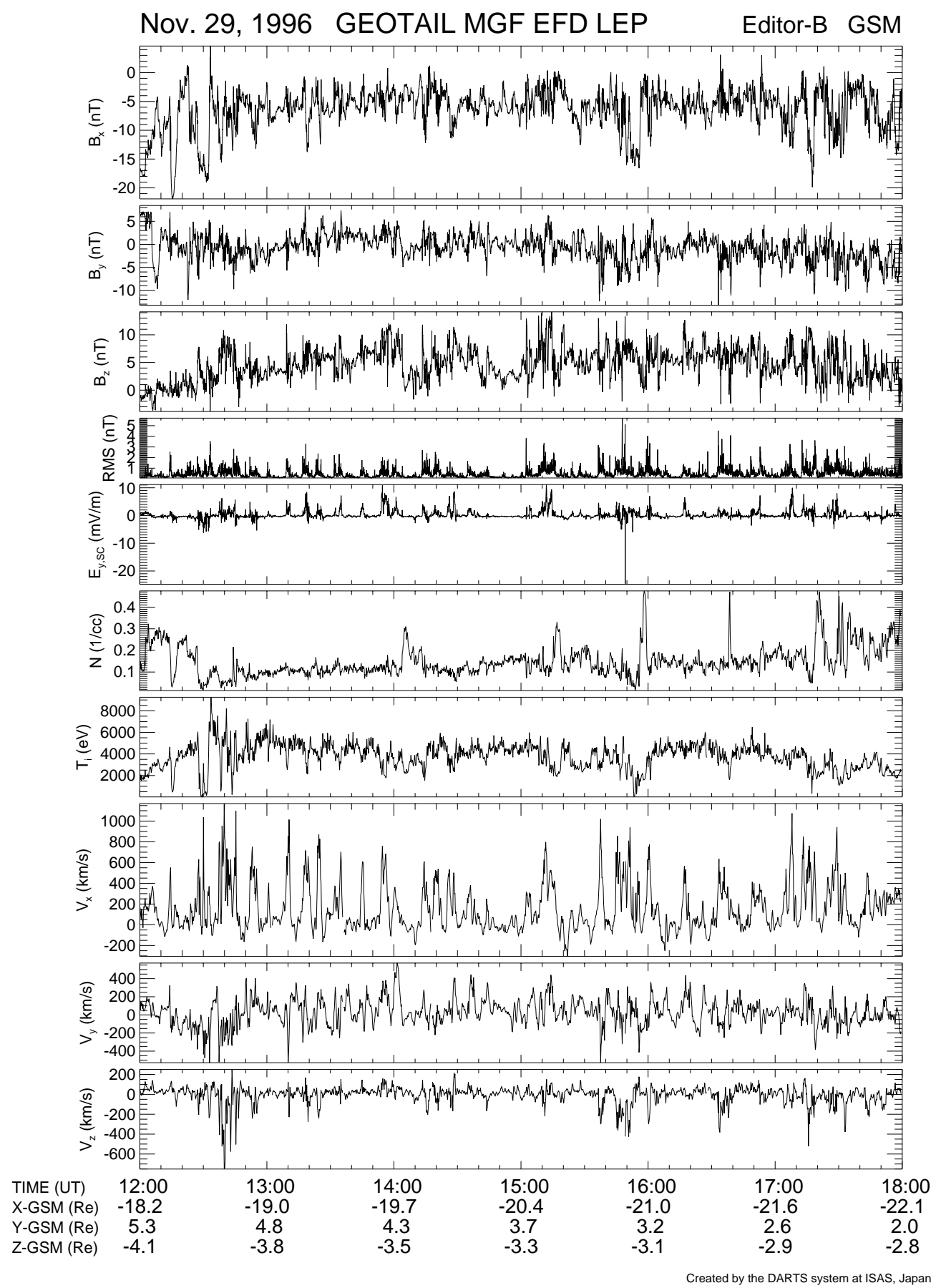

Fig. 1. Overview of the plasma sheet crossing. From top to bottom: three components of magnetic field (in GSM, 3-s data); amplitude of magnetic variations (RMS) in 3-s samples; electric field (3-s); density, temperature and flow velocity of ions (12-s).

Typical power in a spectrum (at the low frequency edge) has a tendency to grow with velocity. In Fig. 4 we compared 16384-point spectra averaged separately for the intervals with the low (the mean $\sim 150 \mathrm{~km} / \mathrm{s}$ ) and the high plasma velocity (the mean $\sim 350 \mathrm{~km} / \mathrm{s}$ ). For the latter the power was the factor of 3-5 higher and the spectrum had more pronounced lower frequency part with the index $\sim 1.6$ and the kink just below $0.1 \mathrm{~Hz}$. For the case with the lower velocity the kink was closer to $0.01 \mathrm{~Hz}$.

The MF part above the kink in Fig. 4 had the index $\sim 2.6$, somewhat larger for the higher spectrum. To reveal possible power dependence of the MF spectral index in more detail, a set of $84 \times 4096$-point ( $\sim 250 \mathrm{~s}$ ) spectra was computed (Table 3 ). The index (in the range $0.07-0.4 \mathrm{~Hz}$ ) also increased 

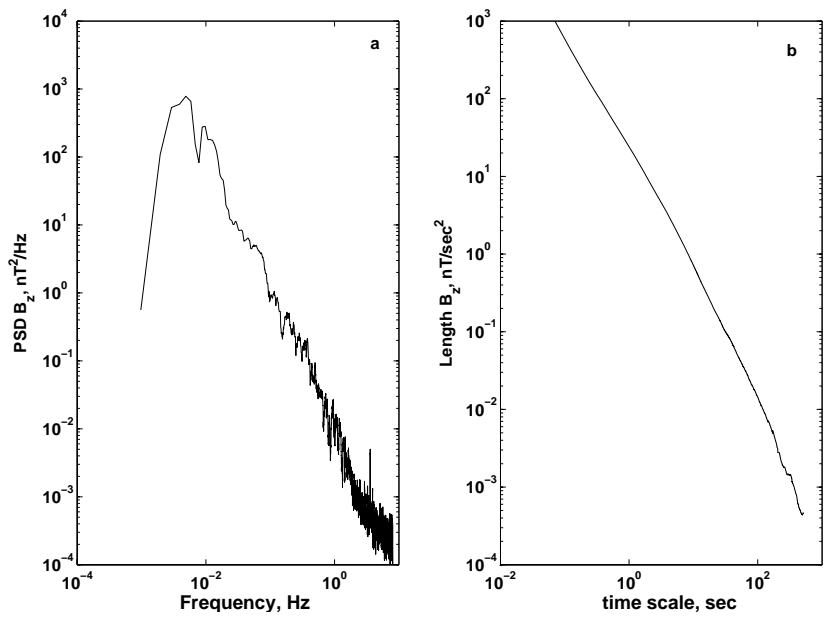

Fig. 2. Sample PSD (left) and length (right) spectra.

with power, but not so drastically as for the lower frequencies (Fig. 3b). The linear regression coefficient between the decimal logarithm of power (in $\mathrm{nT}^{2} / \mathrm{Hz}$ ) and $\alpha$ was 0.28 . There was no significant difference between the components of magnetic field.

Spectra of long intervals average out spiky variations at frequencies above $0.1 \mathrm{~Hz}$. More spatially resolved spectra were computed in $336 \times 1024$-point long $(\sim 64 \mathrm{~s})$ intervals. Several sample spectra are in Fig. 5a. Their amplitudes and slopes vary substantially, and the safe range of the index determination is $0.3-1.1 \mathrm{~Hz}$. According to statistics of Fig. $5 \mathrm{~b}$ the indices were smaller, when amplitudes were below some threshold $\sim 0.04 \mathrm{nT}^{2} / \mathrm{Hz}$ (approximately in $50 \%$ of cases). The average indices and their variance for such higher power cases are in Table 3. The average index $\alpha$ was larger than for $\mathrm{MF}$ and was closer to 3 . The linear regression coefficient between the decimal logarithm of power (in $\mathrm{nT}^{2} / \mathrm{Hz}$ ) and $\alpha$ was 0.72. However it should be noted, that according to Fig. 5a (red and blue curves) spectra with smaller indices were still substantially higher than the noise level.

Finally we checked the Berry relation. The sums $\alpha+2 \delta$ are included in Tables 2-3. In all cases they were larger than 5. The scatter of indices of individual spectra (corresponding to Table 3 statistics) is in Fig. 6a. The LF, MF and HF points occupied distinctly different parameter space, but generally above the Berry line. X, Y, Z components yield similar results. The difference of $\alpha+2 \delta$ from 5 was larger for higher frequencies. Since most of $\alpha+2 \delta$ variability comes from PSD $\alpha$, cases with the lower power generally had smaller $\alpha$ and thus were closer to 5 .

Statistics of indices obtained from the six other data intervals (the data are not shown) is consistent with the results presented above. Here only the average $\alpha$ and $\delta$ in the LF and MF ranges were shown for all seven crossings and three components (Fig. 6b). Average indices were computed using average spectra (one spectrum per interval and component).
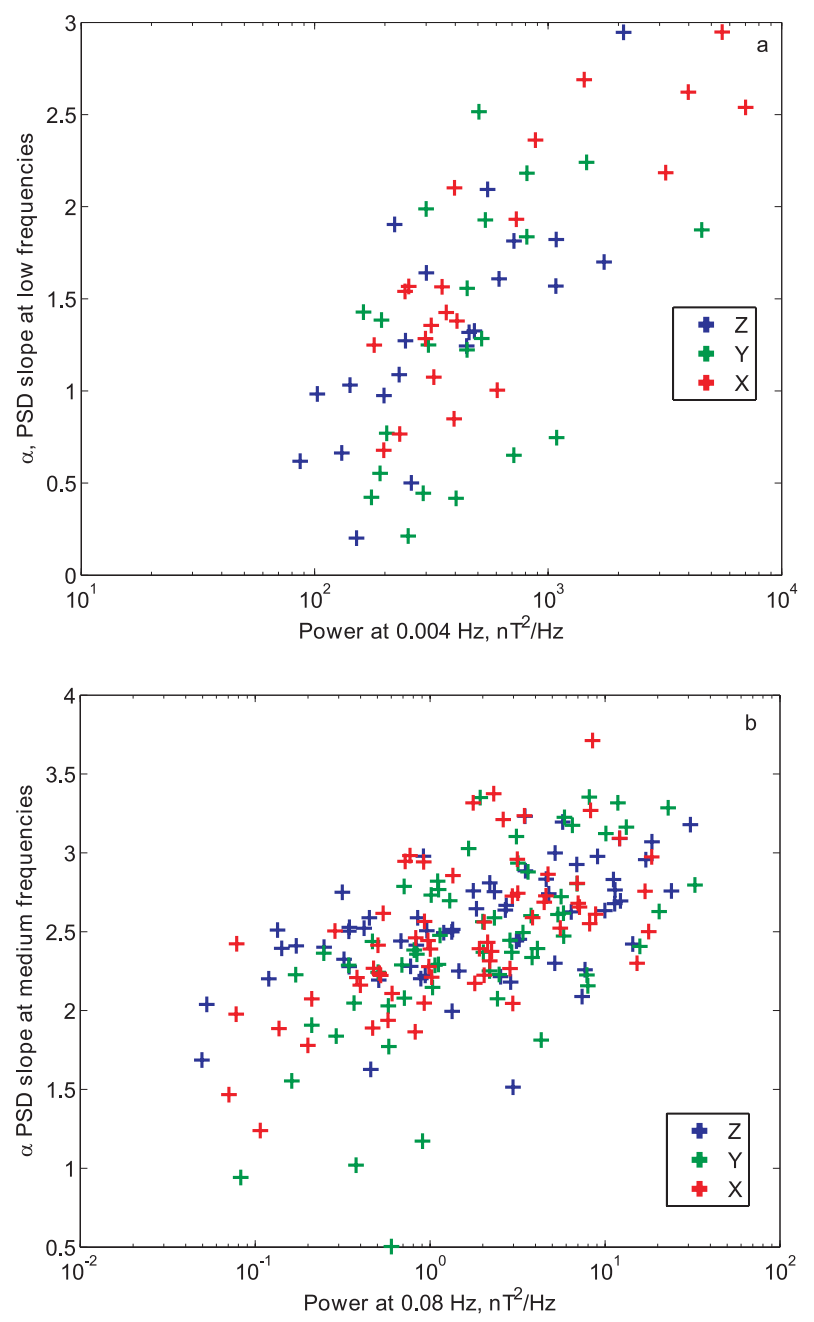

Fig. 3. Relation between power and PSD index for LF (a) and MF (b) ranges.

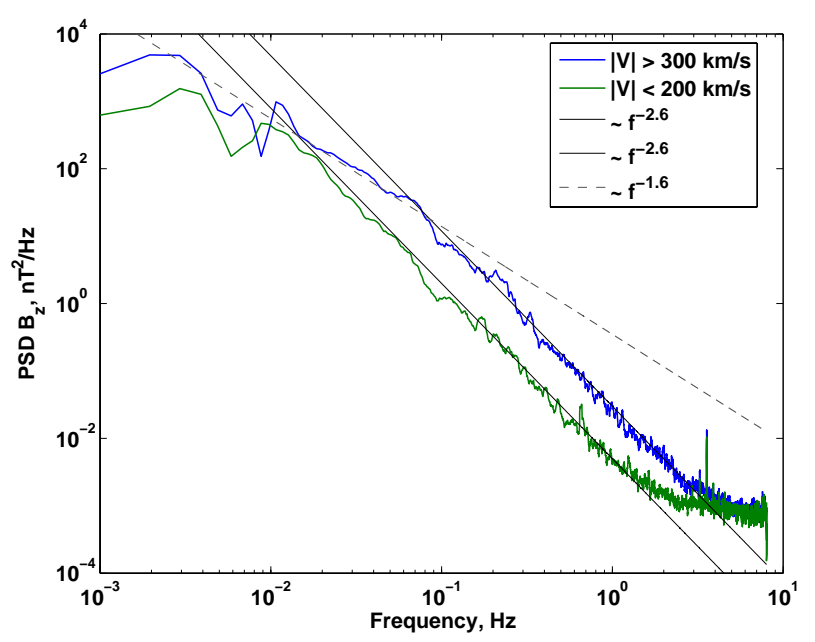

Fig. 4. Averaged PSD spectra for low and high plasma velocity. 

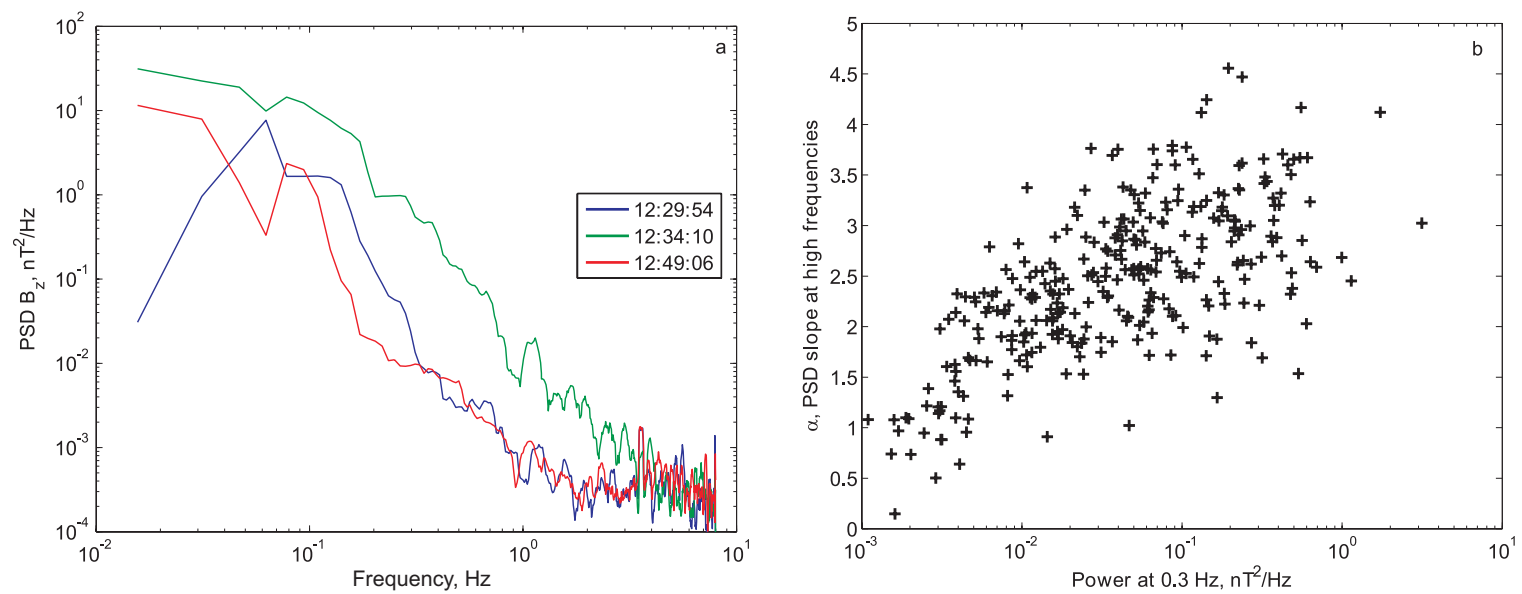

Fig. 5. (a) Sample high frequency spectra. Start times of spectra are specified. (b) Relation between power and PSD index for HF (Z component only).
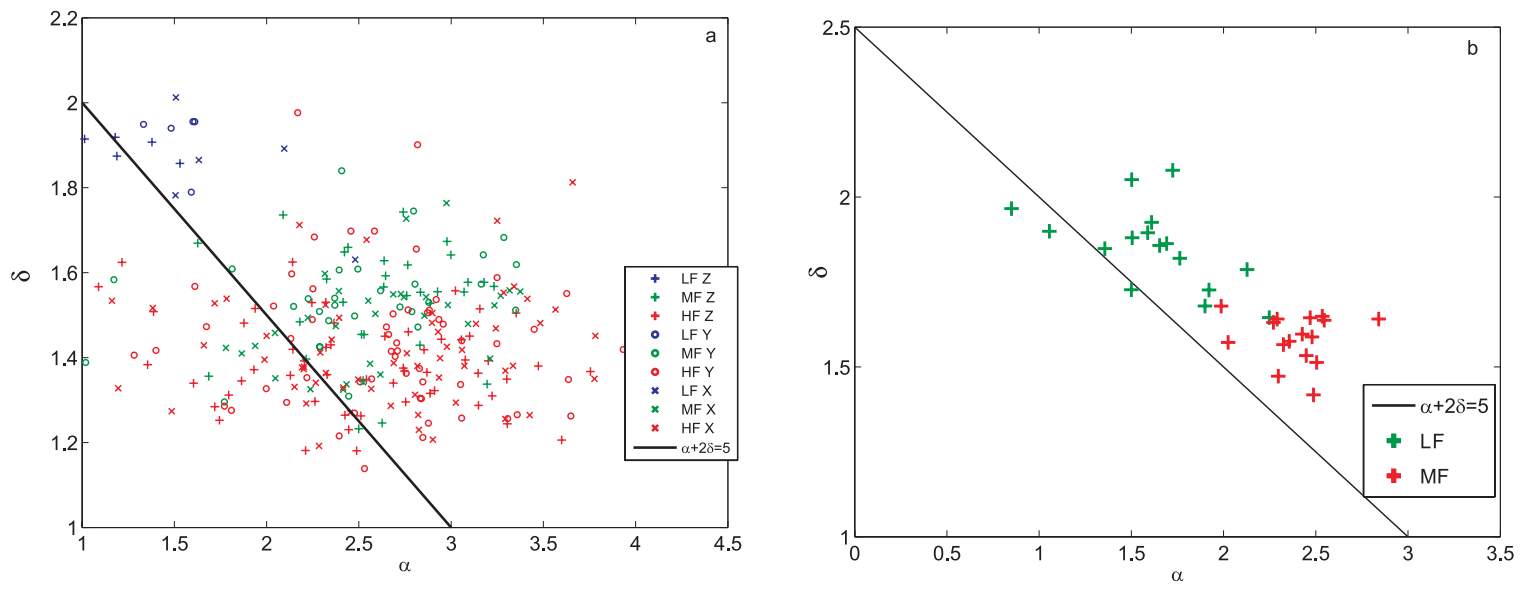

Fig. 6. The scatter of $\alpha$ and $\delta$ indices. Lines denote Berry relation (see text). (a) For the 29 November 96 event (using statistics of Table 3 ). Only every second MF point and every fifth HF point are shown for clarity. (b) Average values for seven events (all three components shown).

The scatter in Fig. 6b was much smaller than in Fig. 6a due to multi-hour averaging and the stable deviation of the Berry relation from 5 is confirmed for both frequency ranges.

\section{Discussion}

Several cases of prolonged observations of the disturbed plasma sheet by Geotail spacecraft helped us to study variations of spectral shapes of low frequency (below $1 \mathrm{~Hz}$ ) magnetic turbulence. Average PSD and length spectra indices as well as kink frequencies are consistent with previous investigations. The average values and their scatter were summarized in Tables 2-3 and Fig. 6. In what follows we discuss our findings for each frequency range.
The most stable spectral characteristics were observed in the medium frequency range $(\sim 0.02-0.2 \mathrm{~Hz})$. Indices of PSD spectra were about $2.4-2.6$ on average and only moderately depended on the wave power. These values were distinctly different from the Kolmogorov (5/3) or Kraichnan $(3 / 2)$ turbulence cascades and were more consistent with the Milovanov and Zelenyi prediction (7/3) (Milovanov et al., 1996; Zelenyi et al., 1998). Stability of structure properties in this range might be due to non-locality of non-adiabatic ion trajectories, which essentially cross the whole tail. Thus the picture is well mixed on the large scales.

Boundary (kink) frequencies of this range vary a lot. Zelenyi et al. (1998) suggested dimensional estimates of $0.01 \mathrm{~Hz}$ and $0.1 \mathrm{~Hz}$ for the lower and the upper bounds, respectively. Kink frequency depends on the Doppler shift (local plasma velocity) and/or local plasma parameters (such as 
ion larmor radius). In the previous investigations rather different kink positions were reported. In our data the lower frequency kink moved from 0.01 to almost $0.1 \mathrm{~Hz}$ (Fig. 4) with the increase of mean ion velocity from 150 to $350 \mathrm{~km} / \mathrm{s}$. Such a shift may happen due to the Doppler shift, and then the typical spatial scale was of about $2000 \mathrm{~km}$. The upper kink frequency between MF and HF ranges is less detectable, since their indices were close.

For investigation of higher frequencies above the upper kink it was important to select short intervals with substantial signal and avoid parts in which the instrument noise dominates. PSD indices in this range depend on power and it is not excluded that lower amplitude (but still substantially above the noise level) fluctuations with the flatter spectra may be due to a different generation mechanism. PSD indices closer to 3 for the higher power cases generally corresponded to the estimate of Zelenyi et al. (1998). It appears more optimal to consider specific generation events (at boundaries and flows) for studies of such variations.

Less straightforward is interpretation of the lowest frequency part. The PSD index here strongly depends on power. This finding is in agreement with the investigation of Vörös et al. (2007), who interpreted the 1.5 scaling extending to approximately $0.1 \mathrm{~Hz}$ as the turbulent cascade during strong flows. Frequency values however might be misleading due to stronger Doppler shifts typical for such events. Contrary to the MF range, LF spectral indices were substantially different for X, Y, Z cartesian components of magnetic field, likely due to different acting mechanisms. At the larger scales variations in $\mathrm{X}$ are dominated by flapping (up and down motion of the whole tail), in $\mathrm{Y}$ - by transient field-aligned currents, while in $\mathrm{Z}$ - by cross-tail current variations.

Scales of variations below $0.01 \mathrm{~Hz}$ are comparable with that of typical plasma sheet reconfigurations (substorms, bursty flows), which substantially change properties and geometry of the plasma sheet. Whether it is reasonable to apply "turbulence" terminology to such changes remains under discussion. From the statistical point of view their detailed identification requires exceptionally long steady intervals, which are essentially rare and might represent some special state of the magnetotail, so that obtained results would be not applicable in other conditions.

Sporadic plasma flows with varying speeds may potentially mix up original frequency ranges or add new fluctuation types with differing spectral indices. However this problem does not affect the most of our conclusions, specifically, calculations of Berry relation. Frequency ranges for index determination were selected rather conservatively to avoid boundaries between ranges in the most of cases.

An important general feature discovered in this investigation is a tendency of PSD indices to grow with power of the signal. It has to be explained with the future research and comparison with models. Fluctuation power is generally known to be positively correlated with appearance of plasma flows. Therefore a part of such index variability might indeed be related to changes and/or shifts of fluctuation modes. However, in our statistics dependence of spectral indices from flow speed clearly emerged only for the LF range (Fig. 4) in consistency with results of Vörös et al. (2007), which were obtained for specially selected interval of constant velocity.

In a standard approach the fractal dimension $\delta$ (power law index of the length spectrum) is coupled with the PSD index via the Berry relation and does not contain additional independent information about the signal. However as it is shown by our investigation, the Berry relation is substantially violated. The deviation of the order of $2 / 3$ (from 5) is comparable with the difference between the indices from the different turbulence models. Thus blind usage of Berry relation might lead to substantial misinterpretation of turbulence properties. Such deviation might be caused by non-Gaussianity of the signal (intermittency).

\section{Conclusions}

Low frequency magnetic field fluctuations in the plasma sheet received considerable attention, because reliable DC magnetic measurements were readily available, and the topic seemed to be quite important for the magnetotail dynamics. Interpretation and quantification of observed waves however, turned out to be a challenging task due to a number of complications, such as entangling of spatial and temporal variations and non-stationarity of the plasma background.

Our investigation with a help of several long observation intervals confirmed typical index values reported in the previous publications. Power spectral density spectra generally followed power laws with two kink frequencies. We now better understand variance of spectra. A part of spectra, likely related with ion kinetic motion, had the most stable indices 2.4-2.6, while below $0.02 \mathrm{~Hz}$ and above $0.2 \mathrm{~Hz}$ the indices strongly varied with fluctuation power and plasma sheet activity. Stable deviations from the Berry relation between PSD index and fractal dimension were revealed. Extensive comparison of these results with different numeric models of turbulence will be very helpful to advance our understanding of the topic.

Acknowledgements. The work was supported in part by the Russian grant HIII-472.2008.2 and INTAS grant 06-1000017-8943. A. A. P. is grateful to L. Zelenyi and Z. Vörös for fruitful discussions. Geotail magnetic field and plasma data were provided by T. Nagai and Y. Saito through DARTS at Institute of Space and Astronautical Science, JAXA in Japan. We also thank Iku Shinohara for the help with the Geotail data access.

Edited by: J. Büchner

Reviewed by: two anonymous referees 


\section{References}

Abry, P., Flandrin, P., Taqqu, M. S., and Veitch, D.: Wavelets for the analysis, estimation and synthesis of scaling data, in: SelfSimilar Network Traffic and Performance Evaluation, edited by: Park, K. and Willinger, W., Wiley (Interscience Division), New York, 39-88, 2000.

Bauer, T. M., Baumjohann, W., Treumann, R. A., Sckopke, N., and Lühr, H.: Low-frequency waves in the near-Earth plasma sheet, J. Geophys. Res., 100, 9605-9618, 1995.

Bendat, J. S. and Piersol, A. G.: Random data. Analysis and measurement procedures, Wiley, 1986.

Borovsky, J. E. and Funsten, H. O.: MHD turbulence in the Earths plasma sheet: Dynamics, dissipation, and driving, J. Geophys. Res., 108(A7), 1284, doi:10.1029/2002JA009625, 2003.

Burlaga, L. F. and Klein, L. W.: Fractal structure of the interplanetary magnetic field, J. Geophys. Res., 91, 347-350, 1986.

Chang, T.: Self-organized criticality, multi-fractal spectra, sporadic localized reconnections and intermittent turbulence in the magnetotail, Phys. Plasmas, 6, 4137-4145, 1999.

Chang, T., Tam, S. W. Y., Wu, C.-C., and Consolini, G.: Complexity, forced and/or self-organized criticality, and topological phase transitions in space plasmas, Space Sci. Rev., 107, 425445, 2003.

Dudok de Wit, T. and Krasnosel'skikh, V. V.: Non-Gaussian statistics in space plasma turbulence: fractal properties and pitfalls, Nonlin. Processes Geophys., 3, 262-273, 1996, http://www.nonlin-processes-geophys.net/3/262/1996/.

Dudok de Wit, T.: Numerical schemes for the analysis of turbulence - a tutorial, in: Space Plasma Simulation, edited by: Büchner, J., Dum, C. and Scholer, M., Springer, Lect. Notes Phys., 615, 315343, 2003.

Gurnett, D. A., Frank, L. A., and Lepping, R. P.: Plasma waves in the distant magnetotail, J. Geophys. Res., 81, 6059-6071, 1976.

Hoshino, M., Nishida, A., Yamamoto, T., and Kokubun, S.: Turbulent Magnetic Field in the Distant Magnetotail: Bottom-Up Process of Plasmoid Formation?, Geophys. Res. Lett., 21, 29352938, 1994.

Kokubun, S., Yamamoto, T., Acuna, M., Hayashi, K., Shiokawa, K., and Kawano, H.: The Geotail magnetic field experiment, J. Geomagn. Geoelectr., 46, 7-21, 1994.

Lui, A. T. Y.: Multiscale phenomena in the near-Earth magnetosphere, J. Atmos. Sol.-Terr. Phy., 64, 125-143, 2002.

Mandelbrot, B.: Fractals: Form, Chance and Dimension, Freeman, New York, 1977.

Mihalov, J. D., Sonett, C. P., and Colburn, D. S.: Reconnection and noise in the geomagnetic tail, Cosmic Electrodynamics, 1, 178204, 1970.

Milovanov, A. V., Zelenyi, L. M., and Zimbardo, G.: Fractal structures and power law spectra in the distant Earth's magnetotail, J. Geophys. Res., 101, 19903-19910, 1996.

Milovanov, A. V., Zelenyi, L. M., Zimbardo, G., and Veltri, P.: Selforganized branching of magnetotail current systems near the percolation threshold, J. Geophys. Res., 106, 6291-6308, 2001.

Mukai, T., Machida, S., Saito, Y., Hirahara, M., Terasawa, T., Kaya, N., Obara, T., Ejiri, M., and Nishida, A.: The low energy particle (LEP) experiment onboard the Geotail satellite, J. Geomagn. Geoelectr., 46, 669-692, 1994.
Ohtani, S., Higuchi, T., Lui, A. T. Y., and Takahashi, K.: Magnetic fluctuations associated with tail current disruption: fractal analysis, J. Geophys. Res., 100, 19135, 1995.

Ohtani, S., Takahashi, K., Higuchi, T., Lui, A. T. Y., Spence, H. E., and Fennell, J. F.: AMPTE/CCE-SCATHA simultaneous observations of substorm-associated magnetic fluctuations, J. Geophys. Res., 103, 4671-4682, 1998.

Petrukovich, A. A.: Low frequency magnetic fluctuations in the plasma sheet: Observations, analysis and interpretation, in: Nonequilibrium phenomena in plasmas, edited by: Sharma, A. S. and Kaw, P. K., Springer, 145-178, 2005.

Russell, C. T.: Noise in the geomagnetic tail, Planet. Space Sci., 20, 1541-1553, 1972.

Sigsbee, K., Cattell, C. A., Moser, F. S., Tsuruda, K., and Kokubun, S.: Geotail observations of low frequency waves from 0.001 to $16 \mathrm{~Hz}$ during the November 24, 1996 Geospace Environment Modelling substorm challenge event, J. Geophys. Res., 106, 435-445, 2001.

Volwerk, M., Glassmeier, K.-H., Runov, A., Baumjohann, W., Nakamura, R., Zhang, T. L., Klecker, B., Balogh, A., and Reme, H.: Kink mode oscillation of the current sheet, Geophys. Res. Lett., 30, 1320, doi:10.1029/2002GL016467, 2003.

Volwerk, M., Vörös, Z., Baumjohann, W., Nakamura, R., Runov, A., Zhang, T. L., Glassmeier, K.-H., Treumann, R. A., Klecker, B., Balogh, A., and Rème, H.: Multi-scale analysis of turbulence in the Earth's current sheet, Ann. Geophys., 22, 25252533, 2004, http://www.ann-geophys.net/22/2525/2004/.

Vörös, Z., Baumjohann, W., Nakamura, R., Runov, A., Zhang, T. L., Volwerk, M., Eichelberger, H. U., Balogh, A., Horbury, T. S., Glaßmeier, K.-H., Klecker, B., and Rème, H.: Multi-scale magnetic field intermittence in the plasma sheet, Ann. Geophys., 21, 1955-1964, 2003, http://www.ann-geophys.net/21/1955/2003/.

Vörös, Z., Baumjohann, W., Nakamura, R., Volwerk, M., Runov, A., Zhang, T. L., Eichelberger, H. U., Treumann, R., Georgescu, E., Balogh, A., Klecker, B., and Reme, H.: Magnetic turbulence in the plasma sheet, J. Geophys. Res., 109, A11215, doi:10.1029/2004JA010404, 2004.

Vörös, Z., Baumjohann, W., Nakamura, R., Runov, A., Volwerk, M., Asano, Y., Jankovičová, D., Lucek, E. A., and Rème, H.: Spectral scaling in the turbulent Earth's plasma sheet revisited, Nonlin. Processes Geophys., 14, 535-541, 2007, http://www.nonlin-processes-geophys.net/14/535/2007/.

Weygand, J. M., Kivelson, M. G., Khurana, K. K., Schwarzl, H. K., Thompson, S. M., McPherron, R. L., Balogh, A., Kistler, L. M., and Goldstein, M. L.: Plasma sheet turbulence observed by Cluster II, J. Geophys. Res., 110, A01205, doi:10.1029/2004JA010581, 2005.

Zelenyi, L. M., Milovanov, A. V., and Zimbardo, G.: Multiscale magnetic structure of the distant Tail: self-consistent fractal approach, in: New Perspectives on the Earth's magnetotail, edited by: Nishida, A., Baker, D. N., and Cowley, S. W. H., AGU, Washington, DC, Geophysical Monograph 105, 321-339, 1998. 